\title{
The ALDH gene superfamily of Arabidopsis
}

\author{
Hans-Hubert Kirch ${ }^{1}$, Dorothea Bartels ${ }^{1}$, Yanling Wei ${ }^{2}$, Patrick S. Schnable ${ }^{2}$ and \\ Andrew J. Wood ${ }^{3}$
}

${ }^{1}$ University of Bonn, Institute of Molecular Physiology and Biotechnology of Plants (IMBIO), Kirschallee1, D-53115 Bonn, Germany
${ }^{2}$ Center for Plant Genomics, Roy J. Carver Co-Laboratory, lowa State University, Ames, IA 50011-3605, USA
${ }^{3}$ Department of Plant Biology, Southern Illinois University - Carbondale, Carbondale, IL 62901-6509, USA

\begin{abstract}
Aldehyde dehydrogenases (ALDHs) represent a protein superfamily of $\mathrm{NAD}(\mathrm{P})^{+}$-dependent enzymes that oxidize a wide range of endogenous and exogenous aliphatic and aromatic aldehydes. The Arabidopsis genome contains 14 unique ALDH sequences encoding members of nine ALDH families, including eight known families and one novel family (ALDH22) that is currently known only in plants. Here, we identify members of the ALDH gene superfamily in Arabidopsis; provide a revised, unified nomenclature for these ALDH genes; analyze the molecular relationship among Arabidopsis ALDH genes and compare them to ALDH genes from other species, including prokaryotes and mammals; and describe the role of ALDHs in cytoplasmic male sterility, plant defense and abiotic stress tolerance.
\end{abstract}

Many biologically important aldehydes are metabolized by the superfamily of $\mathrm{NAD}(\mathrm{P})^{+}$-dependent aldehyde dehydrogenases [aldehyde: $\mathrm{NAD}(\mathrm{P})^{+}$oxidoreductases, EC 1.2.1] [1]. Sequence comparisons among ALDH genes from bacteria, animals and plants have identified three diagnostic amino acid motifs: (i) the ALDH glutamic acid active site signature sequence MELGGNA (PROSITE PS00687); (ii) the Rossmann fold GxGxxG coenzymebinding site; and (iii) the catalytic thiol (PROSITE PS00070) [1-3]. Currently, 555 genes encoding ALDH proteins have been identified throughout all taxa [4]. Aldehydes are intermediates in a range of fundamental biochemical pathways and are generated during the metabolism of carbohydrates, vitamins, steroids, amino acids and lipids $[5,6]$. Aldehydes can also be generated in response to a suite of environmental stresses that perturb metabolism including salinity, dehydration, desiccation, cold and heat shock $[7,8]$. Although they are common biochemical intermediates, many aldehydes are chemically reactive and toxic at physiological concentrations [9]. Active ALDH enzymes represent an important mechanism for the detoxification of aldehydes by oxidation to their corresponding carboxylic acids [6-9].

The ALDH Gene Nomenclature Committee (AGNC) has established specific criteria for cataloguing deduced ALDH protein sequences [1]. Protein sequences that are

Corresponding author: Andrew J. Wood (wood@plant.siu.edu).

Available online 17 July 2004 more than $40 \%$ identical to other previously identified ALDH sequences compose a family, and sequences more than $60 \%$ identical compose a protein subfamily. Protein sequences that are less than $40 \%$ identical would describe a new ALDH protein family. Previous classifications of the ALDH gene superfamily in eukaryotes have identified 21 protein families based upon sequence identity [2,3,10-12]. To date, plant enzymes are represented in $11 \mathrm{ALDH}$ families: ALDH2, ALDH3, ALDH5, ALDH6, ALDH7, ALDH10, ALDH11, ALDH12, ALDH18, ALDH19 and ALDH21. Three protein families are unique to plants (ALDH11, ALDH19 and ALDH21) [12] and one of these is apparently unique to mosses (ALDH21) [13].

The Arabidopsis genome is the only completely sequenced plant genome, and this allows us for the first time to examine the phylogenetic and molecular relationship of all the ALDH genes in a plant species. Based upon nomenclature developed by the AGNC [1-5], we present here a revised, unified nomenclature for the Arabidopsis ALDH genes and describe the most important structural and functional features of the corresponding ALDH protein families. In addition, the unified nomenclature enables a critical view of a functional classification of ALDH enzymatic activities.

\section{Arabidopsis ALDH gene superfamily}

Aldehyde dehydrogenase (ALDH) and ALDH-like DNA sequences were retrieved from The Arabidopsis Information Resource (TAIR, http://www.arabidopsis.org/), Munich Information center for Protein Sequences (MIPS, http://mips.gsf.de/proj/thal/), the US National Center for Biotechnology Information (NCBI, http:// www.ncbi.nlm.nih.gov/) genome and non-redundant expressed sequence tag (EST) databases using BLASTN, PSI-BLAST and BLASTX [14,15]. The searches were conducted using ALDH3I1 (GenBank Accession number AJ306961), ALDH3H1 (GenBank Accession number $\mathrm{AY} 072122)$ and the ALDH active site signature sequence (PROSITE PS00687). Annotated Arabidopsis ALDH open reading frames (ORFs) were verified by comparison to cDNA and EST sequences. These searches resulted in the identification of 14 Arabidopsis sequences that might encode proteins with the diagnostic motifs described above (Table 1). All these ALDH sequences were submitted to 
Table 1. Arabidopsis thaliana aldehyde dehydrogenase gene superfamily

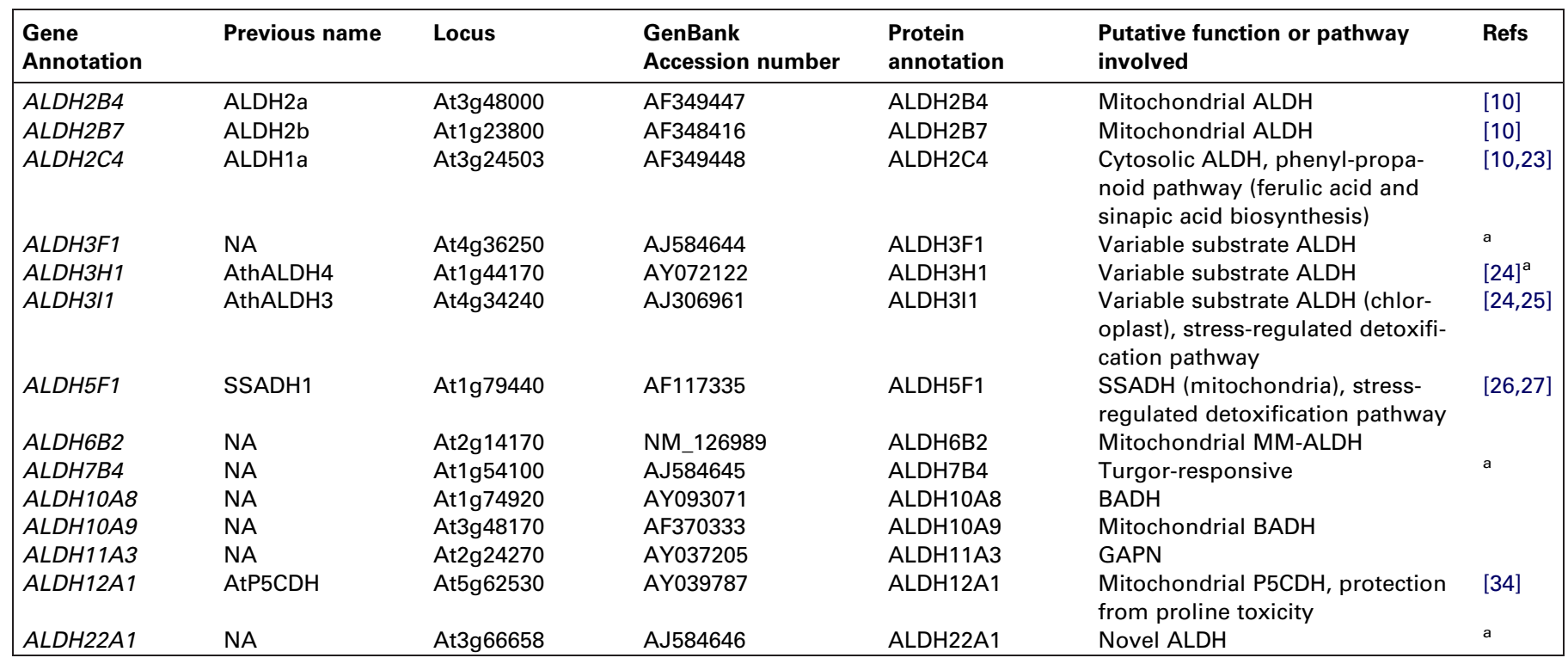

Abbreviations: ALDH, aldehyde dehydrogenase; $\mathrm{BADH}$, betaine aldehyde dehydrogenase; GAPN, non-phosphorylating glyceraldehyde-3-phosphate dehydrogenase; MM-ALDH,

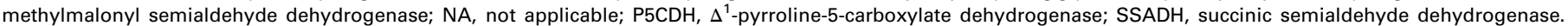
${ }^{\mathrm{a}}$ H.H. Kirch et al., unpublished.

the AGNC [1] and the revised nomenclature presented here has been approved.

The ALDH genes contained in the Arabidopsis genome encode members of nine ALDH protein families - eight previously identified protein families (ALDH2, ALDH3, ALDH5, ALDH6, ALDH7, ALDH10, ALDH11, ALDH12) and one novel protein family (ALDH22) (Table 1, Figure 1). For the classification of each gene family, the root symbol ALDH is followed by the family designation number (1-22), a subfamily designator if required (A, B etc.) and, finally, the individual gene number. For example, the betaine aldehyde dehydrogenase (BADH) encoding cDNA cloned from Spinacia oleracea [16] was renamed using this universal nomenclature as ALDH10A7.

Three of the nine ALDH families in Arabidopsis are represented by more than one gene [family 10 (two members) and families 2 and 3 (three members each)], whereas the remaining six families are encoded by singlecopy genes. It is therefore interesting that no more than four ALDH genes (ALDH6B2, ALDH22A1 and two

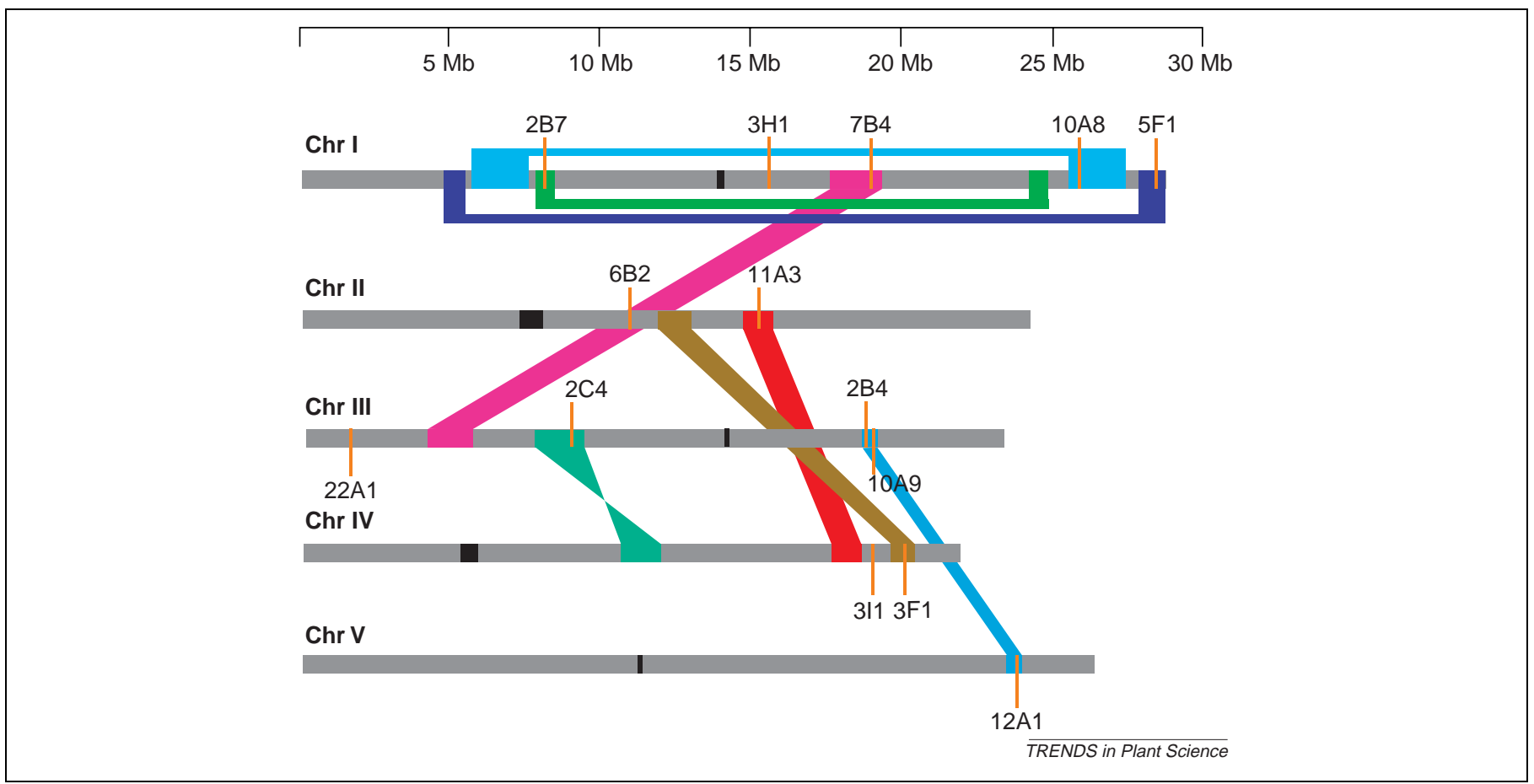

Figure 1. Chromosomal positions of aldehyde dehydrogenase (ALDH) genes in Arabidopsis. Chromosomes I-V (Chr I-V) are depicted as horizontal gray bars with

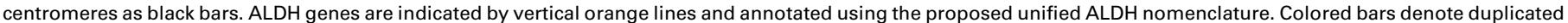

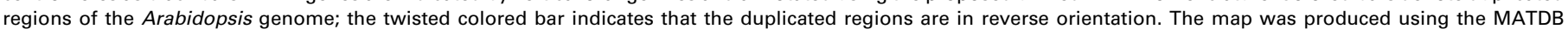
Redundancy Viewer (http://mips.gsf.de/proj/thal/db/gv/gv_frame.html). Adapted from Ref. [37]. 
members of the ALDH3 gene family) are located in nonduplicated regions of the Arabidopsis genome (Figure 1). In addition, only three genes (ALDH2B4, ALDH1OA9 and $A L D H 12 A 1)$ are found within the same duplicated region and thus might represent 'direct' gene duplications, whereas the respective counterparts of the other seven ALDH genes have been lost. Although the three ALDHs show a high protein sequence divergence and even belong to different families, our hypothesis is supported by the fact that the gene contexts around the ALDH genes are highly conserved between the duplicated genomic regions of chromosomes III and V. This implies that functional constraints are responsible for the rapid evolution and sequence divergence of these ALDH genes.

A phylogenetic tree of the Arabidopsis ALDH sequences and other putative plant ALDHs available in the TAIR, MIPS, NCBI genome and non-redundant EST databases is depicted in Figure 2. The root of the tree was placed at the phylogenetically most distantly related ALDH12 family, which shows the least sequence conservation to the rest of the plant ALDHs. The phylogenetic analysis demonstrates that the plant ALDHs split up into ten protein families and confirms the assignment of the Arabidopsis sequences. Although evolutionary relationships could not be clarified for all the different families, the analysis reveals some interesting observations. Family 21, for example, is represented by just one gene from the moss Tortula ruralis, yet it shows a close relationship to the glyceraldehyde-3-phosphate dehydrogenase (GAPDH) family. Similarly, ALDH families 5, 2 and 10 seem to cluster together, and the BADHs (family 10) probably directly diverged from ALDH family 2. Finally, the predicted cytosolic and mitochondrial ALDH forms in family 2 can be clearly separated from each other, which is in accordance with results of a recent characterization of ALDH2 genes from maize and Arabidopsis [10].

\section{Family 2 ALDHs}

Family 2 ALDHs are mitochondrial or cytosolic homotetrameric enzymes and have been extensively studied in humans and yeast $[5,17]$. The first ALDH2 gene identified in plants (rf2) encodes a nuclear restorer of cytoplasmic male sterility (cms) [18,19], and orthologs have been subsequently characterized in maize (Zea mays), rice (Oryza sativa) and Arabidopsis [10,20,21]. Further studies with one of the mitochondrial ALDHs from rice show that this enzyme might be responsible for efficient detoxification of acetaldehyde during re-aeration after submergence of rice plants [22] and suggest a role during ethanolic fermentation for these ALDHs [20-22]. The Arabidopsis genome contains two genes ( $A L D H 2 B 7$ and $A L D H 2 B 4$ ) that encode ALDHs predicted to accumulate in the mitochondria [10]. These proteins, ALDH2B7 and ALDH2B4, are $75 \%$ identical to each other. In vitro activity assays of recombinant proteins indicate that both of these two mitochondrial ALDHs (mtALDHs) can oxidize acetaldehyde and glycolaldehyde, but cannot oxidize L-lactaldehyde, as shown by $E$. coli complementation assays.

Interestingly, all studied plant species, including Arabidopsis, contain genes that encode two highly similar
mtALDHs. Kinetic studies of the two maize mtALDHs (i.e. RF2A and RF2B) indicate that these two enzymes have overlapping, but non-identical substrates. The RF2A protein has a broad substrate spectrum including aliphatic and aromatic aldehydes, whereas the other mtALDH, RF2B, can oxidize only short-chain aliphatic aldehydes [20]. In addition, these two mtALDHs do not accumulate in the same tissues or at the same times [20]. Hence, it appears that plant mtALDHs have undergone functional specialization.

The Arabidopsis genome contains a single gene (ALDH2C4) coding for an ALDH that is predicted to accumulate in the cytosol. This protein, ALDH2C4, exhibits $58 \%$ and $54 \%$ identity to ALDH2B4 and ALDH2B7, respectively, and can oxidize acetaldehyde and glycolaldehyde [10]. In addition, a complementation assay involving an $E$. coli strain that is null for a particular ALDH indicated that this enzyme can also oxidize L-lactaldehyde. More recently, Ramesh B. Nair et al. [23] demonstrated that the reduced epidermal fluorescence1 (ref1) mutant of Arabidopsis is caused by a mutation in the $\mathrm{ALDH} 2 \mathrm{C} 4$ gene and that ALDH2C4 is involved in the oxidization of sinapaldehyde and coniferaldehyde.

\section{Family 3 ALDHs}

In contrast to the mitochondrial and cytosolic ALDH2 family, ALDHs belonging to family 3 are dimeric enzymes located in cytosolic and microsomal fractions. They have been characterized in detail in humans and are associated with carcinogenesis and severe genetic disorders [5]. The first plant ALDH3 gene, $C p-A L D H$, was isolated from the resurrection plant Craterostigma plantagineum in an attempt to identify genes that are crucial for the abscisic acid (ABA) dependent stress response [24]. The Arabidopsis genome contains three genes (ALDH3F1, $A L D H 3 I 1$ and $A L D H 3 H 1$ ) with $60-76 \%$ amino acid identity to $C p-A L D H$. Sequence analysis of ALDH3I1 predicts a putative chloroplast-targeting peptide of 60 amino acids, whereas ALDH3F1 and ALDH3H1 are probably localized to the cytosol.

$A L D H 3 I 1, A L D H 3 H 1$ and $A L D H 3 F 1$ differ in their expression patterns. Similar to $C p-A L D H, A L D H 3 I 1$ expression is induced after exogenous ABA application, high salinity, dehydration and exposure to heavy metals, $\mathrm{H}_{2} \mathrm{O}_{2}$ and paraquat (methyl viologen, which binds to thylakoid membranes and causes the formation of superoxide radicals), suggesting a possible role in response to oxidative stress [24,25]. Interestingly, stress-regulated expression of $A L D H 3 I 1$ is restricted to leaves and the transcript is almost undetectable in roots. By contrast, $A L D H 3 H 1$ is constitutively expressed at a low level in leaves but is activated in response to osmotic stress or after $\mathrm{ABA}$ treatment in root tissue, whereas $A L D H 3 F 1$ expression is not stress responsive at all (H.H. Kirch et al., unpublished). These results indicate that the Arabidopsis ALDH3 gene family members might have evolved as a consequence of functional specialization in different tissues and subcellular compartments.

Kinetic data indicated that Cp-ALDH might be involved in the detoxification of reactive aldehyde species 


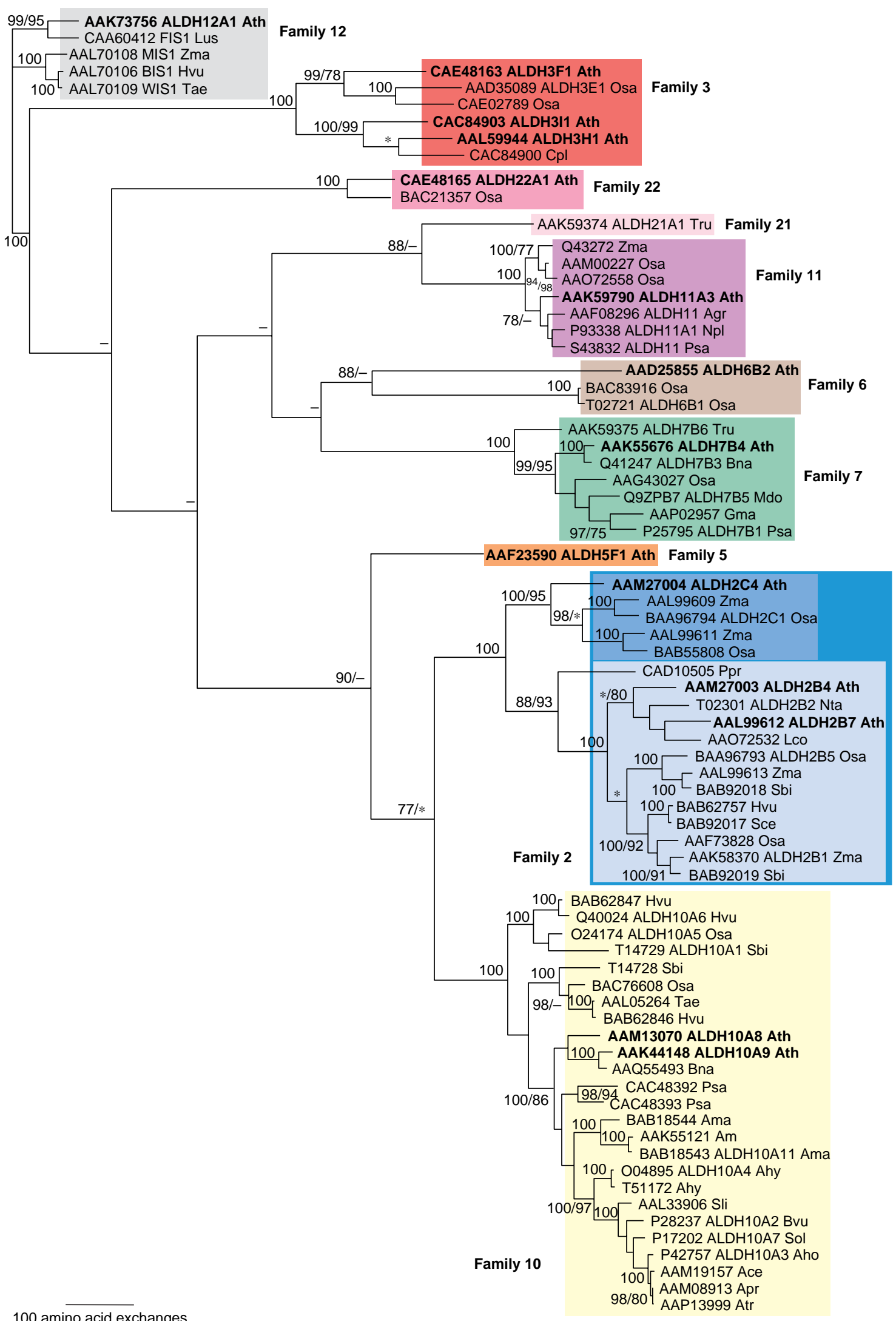

100 amino acid exchanges

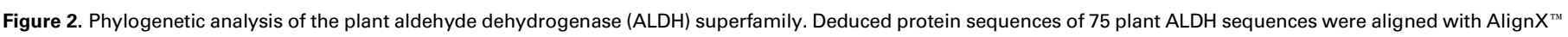

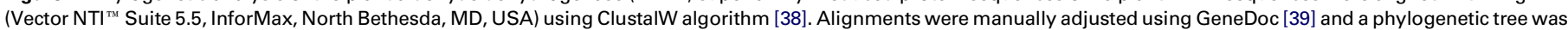

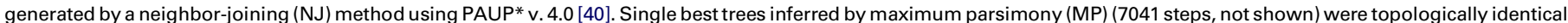

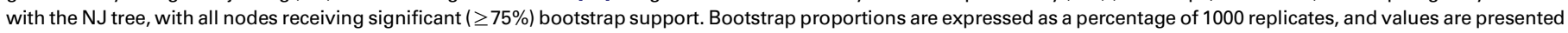

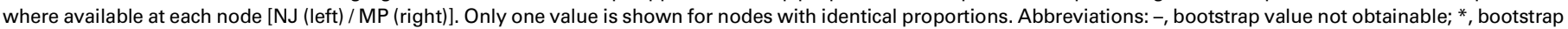


generated by oxidative-stress-associated lipid peroxidation. A functional analysis of ALDH3I1 suggests that this protein is probably involved in a detoxification pathway in plants that limits aldehyde accumulation and oxidative stress [25]. Transgenic Arabidopsis plants ectopically expressing the ALDH3I1 cDNA exhibit an improved tolerance in response to dehydration, salinity $(\mathrm{NaCl})$, heavy metals $\left(\mathrm{Cu}^{2+}\right.$ and $\left.\mathrm{Cd}^{2+}\right), \mathrm{H}_{2} \mathrm{O}_{2}$ and paraquat under laboratory conditions. This tolerance is correlated with a decreased accumulation of lipid-peroxidation-derived reactive aldehydes compared with wildtype plants.

\section{Succinic and methylmalonyl semialdehyde dehydrogenases (ALDH families 5 and 6)}

The Arabidopsis genome contains one succinic semialdehyde dehydrogenase (SSADH; EC 1.2.1.24) gene, $A L D H 5 F 1$, which encodes a protein of 528 amino acids (GenBank Accession number AAF23590; Table 1). To date, $A L D H 5 F 1$ is the only identified member of the succinic semialdehyde family in plants (Figure 2). Analysis of the Arabidopsis database indicates that a second SSADH 'isoform' might exist, encoding a protein of 509 amino acids (NCBI Accession number AAD30232), with 35 amino acids missing at the $\mathrm{N}$-terminus and an insertion of 16 amino acids at position 86 , which could be produced by alternative splicing. However, we have not included this protein in our report here because available cDNA and EST sequences do not support this possibility. SSADHs are involved in $\gamma$-aminobutyric acid (GABA) metabolism as part of the 'shunt' from Glu to GABA [26]. The Arabidopsis protein is localized to mitochondria and a kinetic analysis showed that the recombinant enzyme was specific for succinic semialdehyde and regulated by adenine nucleotides. T-DNA knockout mutants of $A L D H 5 F 1$ result in dwarfed plants with necrotic lesions and are sensitive to both ultraviolet-B light and heat stress [27]. Plants with ssadh mutations accumulate elevated levels of $\mathrm{H}_{2} \mathrm{O}_{2}$, suggesting a role for this gene in plant defense against environmental stress by preventing the accumulation of reactive oxygen species.

The Arabidopsis genome contains a single gene, $A L D H 6 B 2$, that encodes a methylmalonyl semialdehyde dehydrogenase (MM-ALDH, EC 1.2.1.27; Table 1). MM-ALDHs are involved in the degradation of valine to propionyl CoA [28]. However, this enzyme has not been extensively studied in plants and the expression of $A L D H 6 B 2$ has not been investigated.

Stress-associated ALDHs (ALDH families 7, 10, 11 and 12) Several osmotic-stress-inducible ALDH genes have been identified in plants and they compose the majority of the protein families ALDH7, ALDH10, ALDH11 and ALDH12 [12]. Arabidopsis has a single gene, $A L D H 7 B 4$, that encodes a 'turgor-responsive' [29] or 'stress aldehyde' ALDH [13]. It is postulated that ALDH7 gene products are involved in an unknown adaptive metabolic pathway. In angiosperms, ALDH7B homologs are induced by a range of stresses including dehydration, low temperature, heat shock and high concentrations of ABA [12]. Expression analysis of $A L D H 7 B 4$ from Arabidopsis also indicates a strong induction by abiotic stress treatments and after application of ABA to whole plants and to roots (H.H. Kirch et al., unpublished). In sharp contrast to this, $A L D H 7 B 6$ from the moss $T$. ruralis is constitutively expressed in response to $\mathrm{NaCl}$, ultraviolet-C, $\mathrm{ABA}$ and desiccation [13]. In this respect, it is interesting that the moss ALDH is phylogenetically clearly separated from all other plant ALDH7 sequences (Figure 2).

The Arabidopsis genome encodes two members of the ALDH10 protein family, ALDH10A8 and ALDH10A9, which are putative dehydration- and salt-inducible $\mathrm{BADHs}$ (EC 1.2.1.8) that catalyze the oxidation of betaine aldehyde to the compatible solute glycine betaine [16]. The ability to synthesize and/or accumulate glycine betaine is a ubiquitous adaptation to osmotic stress present in bacteria, animals and plants [30].

The Arabidopsis genome contains a single gene, $A L D H 11 A 3$, that encodes a non-phosphorylating GAPDH (GAPN, EC 1.2.1.9). GAPN operates in the cytosol of autotrophic eukaryotes, where it generates NADPH for biosynthetic processes from photosynthetic glyceraldehyde-3-phosphate exported from the chloroplast by the phosphate translocator. ALDH11 catalyzes one of the classic glycolytic 'bypass' reactions unique to plants [31]. Although the role of this enzyme is unclear, Zhifang Gao and Wayne H. Loescher [32] have established that GAPN is the main source of NADPH for mannitol biosynthesis in celery. Expression of ALDH11A3 from Arabidopsis has not been investigated.

A mitochondrial $\Delta^{1}$-pyrroline-5-carboxylate dehydrogenase (P5CDH, EC 1.5.1.12; Table 1), a key enzyme in the degradation of proline to glutamate [33], is encoded by a single gene, $A L D H 12 A 1$, in Arabidopsis. ALDH12A1 was identified by complementing the yeast $\Delta$ put 2 mutant, and its transcript is induced by exogenous proline application and salinity [34].

\section{Novel ALDH (ALDH family 22)}

The novel ALDH22 family is so far represented by three plant genes. One of them (ALDH22A1) was identified in the Arabidopsis genome and encodes a putative aldehyde dehydrogenase of 596 amino acids. Our analyses of rice [35] and maize [36] genome assemblies revealed the

value less than 75\%. Members of each ALDH family are highlighted by different colors: gray, family 12 (P5CDH); red, family 3 (variable substrate; class 3 ALDHs); pink, family 22

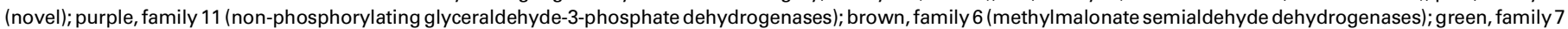

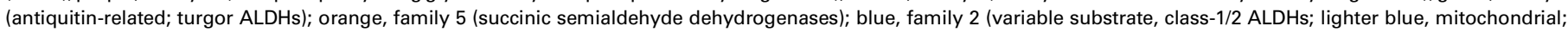

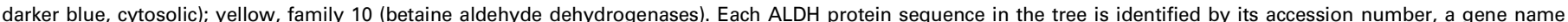

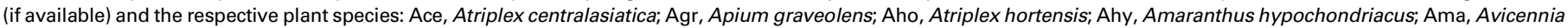

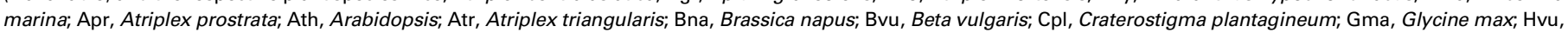

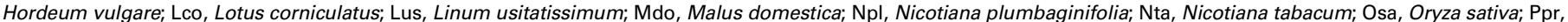

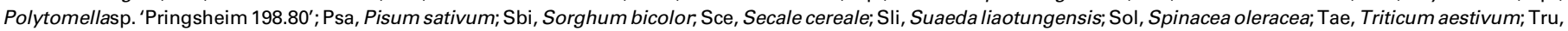
Tortula ruralis; Zma, Zea mays. 
presence of orthologs in both species. The rice ortholog is predicted to encode a closely related protein consisting of 597 amino acids (77\% identity, $89 \%$ similarity; Figure 2). It is not possible to determine the predicted size of the maize ortholog because only partial genomic and cDNA sequences are available. An initial examination of the ALDH22A1 transcript accumulation pattern indicates a low constitutive expression, suggesting that the expression of this ALDH gene is not modulated by abiotic stress (H.H. Kirch et al., unpublished).

\section{Conclusions}

Under non-stress conditions, ALDHs are involved in fundamental plant metabolism and maintain aldehyde concentrations at nontoxic levels. As abiotic stresses perturb metabolism, ALDHs detoxify stress-generated aldehydes and help maintain the balance of the pool of reducing equivalents. The expression of ALDH-related genes seems to be a common 'stress response' within several divergent plant species from mosses to angiosperms and suggests the existence of common mechanisms for the regulation of their genes, emphasizing the conservation of physiological features in response to osmotic stress.

The Arabidopsisgenome contains 14 genes belonging to the ALDH gene superfamily, encoding members of nine distinctive protein families, many of which are associated with stress conditions that limit plant growth and development. One research goal will be to establish that ALDH genes encode authentic enzymatic activity against aldehydes and to determine the endogenous substrate(s) and co-enzyme preference (e.g. $\mathrm{NAD}^{+}$or $\mathrm{NADP}^{+}$) for each protein family. A second goal will be to use the suite of molecular, genetic and genomic tools available in Arabidopsis to identify and characterize the biochemical pathways associated with each protein and/or protein family. Elucidation of the ALDH functions will represent an important step towards understanding basic aspects of osmotic stress responses in plants.

\section{Acknowledgements}

Our work was supported in part by grants to A.J.W. (USDA NRI 97-35100), H.H.K. and D.B. (DFG-AFGN \#BA 712/3-1), and P.S.S. (USDA NRI 00-0347 and 02-01419). We thank Vasilis Vasiliou (University of Colorado Health Sciences Center, Denver, CO) for helpful discussions.

\section{References}

1 Vasiliou, V. et al. (1999) Eukaryotic aldehyde dehydrogenase (ALDH) genes: human polymorphisms, and recommended nomenclature based on divergent evolution and chromosomal mapping. Pharmacogenetics $9,421-434$

2 Perozich, J. et al. (1999) Relationships within the aldehyde dehydrogenase extended family. Protein Sci. 8, 137-146

3 Perozich, J. et al. (1999) The big book of aldehyde dehydrogenase sequences: an overview of the extended family. In Advances in Experimental Medicine and Biology (Vol. 463) (Weiner, H. et al.,eds.), pp. 1-7, Kluwer

4 Sophos, N.A. and Vasiliou, V. (2003) Aldehyde dehydrogenase gene superfamily: the 2002 update. Chem. Biol. Interact. 143-144, 5-22

5 Yoshida, A. et al. (1998) Human aldehyde dehydrogenase gene family. Eur. J. Biochem. 251, 549-557

6 Vasiliou, V. et al. (2000) Role of aldehyde dehydrogenases in endogenous and xenobiotic metabolism. Chem. Biol. Interact. 129, 1-19
7 Bartels, D. (2001) Targeting detoxification pathways: an efficient approach to obtain plants with multiple stress tolerance? Trends Plant Sci. 6, 284-286

8 Barclay, K.D. and Mckersie, B.D. (1994) Peroxidation reactions in plant membranes - effects of free fatty acids. Lipids $29,877-882$

9 Lindahl, R. (1992) Aldehyde dehydrogenases and their role in carcinogenesis. Crit. Rev. Biochem. Mol. Biol. 27, 283-335

10 Skibbe, D. et al. (2002) Characterization of the aldehyde dehydrogenase gene families of Zea mays and Arabidopsis. Plant Mol. Biol. 48, 751-764

11 Sophos, N.A. et al. (2001) Aldehyde dehydrogenase gene superfamily: the 2000 update. Chem. Biol. Interact. 130-132, 323-337

12 Wood, A.J. and Krayesky, S. (2002) The role of aldehyde dehydrogenases (ALDHs) in plant stress tolerance. In Biochemical \& Molecular Responses of Plants to the Environment (Wood, A.J. ed.), pp. 1-14, Research Signpost

13 Chen, X. et al. (2002) The stress-responsive gene ALDH21A1 describes a novel eukaryotic aldehyde dehydrogenase protein family. J. Plant Physiol. 159, 677-684

14 Altschul, S.F. et al. (1990) Basic local alignment search tool. J. Mol. Biol. 215, 403-410

15 Altschul, S.F. et al. (1997) Gapped BLAST and PSI-BLAST: a new generation of protein database search programs. Nucleic Acids Res. 25, 3389-3402

16 Weretilnyk, E.A. and Hanson, A.D. (1990) Molecular cloning of a plant betaine aldehyde dehydrogenase, an enzyme implicated in adaptation to salinity and drought. Proc. Natl. Acad. Sci. U. S. A. 87, 2745-2749

17 Navarro-Aviño, J.P. et al. (1999) A proposal for nomenclature of aldehyde dehydrogenases in Saccharomyces cerevisiae and characterization of the stress-inducible $A L D 2$ and $A L D 3$ genes. Yeast 15, 829-842

18 Ciu, X.Q. et al. (1996) The RF2 nuclear restorer gene of male-sterile, T-cytoplasm maize. Science 272, 1334-1336

19 Liu, F. et al. (2001) Mitochondrial aldehyde dehydrogenase activity is required for male fertility in maize (Zea mays L.). Plant Cell 13, 1063-1078

$20 \mathrm{Liu}$, F. and Schnable, P.S. (2002) Functional specialization of maize mitochondrial aldehyde dehydrogenases. Plant Physiol. 130, $1657-1674$

21 Nakazono, M. et al. (2000) Expression of a gene encoding mitochondrial aldehyde dehydrogenase in rice increases under submerged conditions. Plant Physiol. 124, 587-598

22 Tsuji, H. et al. (2003) Induction of mitochondrial aldehyde dehydrogenase by submergence facilitates oxidation of acetaldehyde during re-aeration in rice. FEBS Lett. 546, 369-373

23 Nair, R. et al. (2004) The Arabidopsis thaliana REDUCED EPIDERMAL FLUORESCENCE1 gene encodes an aldehyde dehydrogenase involved in ferulic acid and sinapic acid biosynthesis. Plant Cell 16, $544-554$

24 Kirch, H.H. et al. (2001) Novel ABA- and dehydration-inducible aldehyde dehydrogenase genes isolated from the resurrection plant Craterostigma plantagineum and Arabidopsis thaliana. Plant J. 28, $555-568$

25 Sunkar, R. et al. (2003) Overexpression of a stress-inducible aldehyde dehydrogenase gene from Arabidopsis thaliana in transgenic plants improves stress tolerance. Plant J. 35, 452-464

26 Bouché, N. and Fromm, H. (2004) GABA in plants: just a metabolite? Trends Plant Sci. 9, 110-115

27 Bouché, N. et al. (2003) Mitochondrial succinic-semialdehyde dehydrogenase of the gamma-aminobutyrate shunt is required to restrict levels of reactive oxygen intermediates in plants. Proc. Natl. Acad. Sci. U. S. A. 100, 6843-6849

28 Steele, M.I. et al. (1992) Characterization of the $m m s A B$ operon of Pseudomonas aeruginosa PAO encoding methylmalonate-semialdehyde dehydrogenase and 3-hydroxyisobutyrate dehydrogenase. J. Biol. Chem. 267, 13585-13592

29 Guerrero, F.D. et al. (1990) Turgor-responsive gene transcription and RNA levels increase rapidly when pea shoots are wilted. Plant Mol. Biol. 15, 11-26

30 Rhodes, D. and Hanson, A.D. (1993) Quaternary ammonium and tertiary sulphonium compounds in high plants. Annu. Rev. Plant Physiol. Plant Mol. Biol. 44, 357-384 
31 Plaxton, W. (1996) The organization and regulation of plant glycolysis. Annu. Rev. Plant Physiol. Plant Mol. Biol. 47, 185-214

32 Gao, Z. and Loescher, W.H. (2000) NADPH supply and mannitol biosynthesis. Characterization, cloning, and regulation of the nonreversible glyceraldehyde-3-phosphate dehydrogenase in celery leaves. Plant Physiol. 124, 321-330

33 Hare, P.D. and Cress, W.A. (1997) Metabolic implications of stressinduced proline accumulation in plants. Plant Growth Regul. 21, 79-102

34 Deuschle, K. et al. (2001) A nuclear gene encoding mitochondrial delta-pyrroline-5-carboxylate dehydrogenase and its potential role in protection from proline toxicity. Plant J. 27, 345-356

35 Yuan, Q.P. et al. (2003) The TIGR rice genome annotation resource: annotating the rice genome and creating resources for plant biologists. Nucleic Acids Res. 31, 229-233
36 Emrich, S.J. et al. (2004) A strategy for assembling the maize (Zea mays L.) genome. Bioinformatics 20, 140-147

37 The Arabidopsis Genome Initiative. (2000) Analysis of the genome sequence of the flowering plant Arabidopsis thaliana. Nature 408, $796-815$

38 Thompson, J.D. et al. (1994) CLUSTAL W: improving the sensitivity of progressive multiple sequence alignment through sequence weighting, position-specific gap penalties and weight matrix choice. Nucleic Acids Res. 22, 4673-4680

39 Nicholas, K.B. et al. (1997) GeneDoc; analysis and visualization of genetic variation. EMBnet News 4, 14 (http://www.hgmp.mrc.ac.uk/ embnet.news/)

40 Swofford, D.L. (2002) PAUP*: Phylogenetic Analysis Using Parsimony (and Other Methods) 4.0 Beta, Sinauer Associates, Sunderland, MA, USA

\section{NEW IMPACT FACTOR}

A big Thank You from the editorial team of Trends in Plant Science to all our loyal readers. Thanks to you, the 2003 ISI impact factor for Trends in Plant Science is 13.4, ensuring our rank as the No. 1 monthly review journal in the Plant Science subject area.

The top five cited articles included in the current impact factor are:
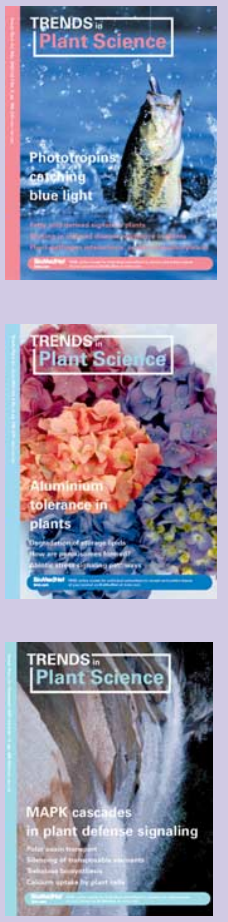

Susanne Brink (Editor)

Caroline Woods (Assistant Editor)

Catherine Hughes (Editorial Coordinator)

\section{Phototropins 1 and 2: versatile plant blue-light receptors} Winslow R. Briggs and John M. Christie. Trends in Plant Science (2002) Vol. 7 (5) 204-210

Nitric oxide: comparative synthesis and signaling in animal and plant cells David Wendehenne, Alain Pugin, Daniel F. Klessig and Jörg Durner Trends in Plant Science (2001) Vol. 6 (4) 177-183

Aluminium tolerance in plants and the complexing role of organic acids Jian Feng Ma, Peter R. Ryan and Emmanuel Delhaize Trends in Plant Science (2001) Vol. 6 (6) 273-278

Oxidative stress, antioxidants and stress tolerance

Ron Mittler

Trends in Plant Science (2002) Vol. 7 (9) 405-410

MAPK cascades in plant defense signaling Shuqun Zhang and Daniel F. Klessig

Trends in Plant Science (2001) Vol. 6 (11) 520-527 